\title{
Síndrome de Wallenberg como forma de presentación de un angioma cavernoso
}

\author{
P. Cardona-Portela, A. Escrig-Avellaneda, F. Rubio-Borrego
}

\section{WALLENBERG'S SYNDROME AS THE PRESENTING SYMPTOM OF A CAVERNOUS ANGIOMA}

\begin{abstract}
Summary. Introduction. Cavernous angiomas account for 5-13\% of all vascular malformations. In $75 \%$ of cases they are situated in the posterior fossa and up to $30 \%$ are associated with abnormal venous drainage. The main complication is haemorrhage; the presence of a neurological focus without radiological evidence of bleeding is very rare. Case report. We report the case of a 54-year-old male with cardiovascular risk factors who presented symptoms that progressively deteriorated over a 72-hour period involving the left lower cranial nerves, sensory impairment and coordination disorder, compatible with Wallenberg's syndrome. Two computerised axial tomography scans of the brain were normal and so a tentative diagnosis of ischemic stroke in progression was proposed. Five days later, magnetic resonance imaging (MR) revealed the presence of a venous angioma and associated abnormal venous drainage. Conclusions. Cavernous angiomas present a dynamic balance between intracavernous bleeding and thrombosis, with very slow venous blood flow. Upsetting this balance leads to an increase in the intracavernous pressure and involvement of the surrounding tissue, with no radiological expression of bleeding. In these cases MR scanning helps to distinguish between a vascular malformation with reduced blood flow and a clinical picture of ischemic stroke of an arterial origin. [REV NEUROL 2004; 39: 837-40]
\end{abstract}

Key words. Anomalous venous drainage. Cavernous angiomas. Stroke. Venous angiomas. Wallenberg.

\section{INTRODUCCIÓN}

Los angiomas cavernosos representan el 5-13\% del total de malformaciones vasculares cerebrales. Aunque su prevalencia es del 0,5-1\% del total de necropsias, la mayoría cursan de forma asintomática o con síntomas neurológicos sin riesgo vital para el paciente, por lo que se han considerado como un tipo de malformación vascular con una evolución y pronóstico benigno. En el $25 \%$ de los pacientes se encuentran asociados a casos familiares en relación con el cromosoma 7, y éstos presentan un mayor número de lesiones y un curso clínico más tormentoso.

El 75\% de los cavernomas se encuentra en el territorio supratentorial, y el $25 \%$ en el cerebelo y en el tronco; en este último es más frecuente en la protuberancia [1,2]. Los de localización infratentorial, tanto por su curso clínico [3] como por su recurrencia, parecen diferenciarse de los del territorio supratentorial, por lo que deberían reconocerse como un subgrupo de cavernomas. En los cavernomas de la región del tronco cerebral, si bien la hemorragia es la complicación más importante, el inicio clínico en forma de focalidad neurológica sin signos de sangrado en las pruebas de imagen es una complicación que puede pasar desapercibida [4,5].

\section{CASO CLÍNICO}

Paciente varón de 54 años hipertenso, dislipémico, fumador de dos paquetes al día y alcoholismo moderado; con antecedentes de cardiopatía isquémica y enfermedad de tres vasos, tratado con angioplastia transluminal percutánea y endoprótesis hace dos años por infarto inferolateral. Hace un año se realizó una angioplastia por estenosis aortoilíaca (se desestimó realizar una derivación por ser una intervención de alto riesgo debido a la cardiopatía). Se encuentra en tratamiento antiagregante con $300 \mathrm{mg} /$ día de aspirina.

Acudió a urgencias por un cuadro de inestabilidad y disartria de dos días de evolución. En la exploración presentaba síndrome de Horner izquierdo,

Recibido: 04.02.04. Aceptado tras revisión externa sin modificaciones: 12.05.04. Servicio de Neurología. Hospital de Bellvitge. L'Hospitalet de Ll., Barcelona, España.

Correspondencia: Dr. Pedro Cardona Portela. Servicio de Neurología. Hospital de Bellvitge. Feixa Llarga, s/n. E-08907 L'Hospitalet de Ll. (Barcelona).E-mail: 34109pcp@comb.es

(C) 2004, REVISTA DE NEUROLOGÍA con hipoestesia braquiocrural derecha e hipoestesia facial izquierda en las dos últimas ramas trigeminales, junto con dismetría izquierda con inestabilidad grave en la marcha, acompañada de hipo y leve parálisis facial derecha.

La analítica de urgencias presentaba bioquímica, hematología y coagulación normal. La radiografía de tórax revelaba cardiomegalia y el electrocardiograma (ECG) mostraba signos de infarto antiguo en la cara lateral e inferior, con ritmo sinusal pero con frecuentes extrasístoles supraventriculares; la tomografía axial computarizada (TAC) craneal fue normal (Fig. 1a). Se ingresó al paciente con tratamiento antiagregante con clopidogrel y el cuadro clínico se orientó como síndrome de Wallenberg por un posible infarto lateral del bulbo izquierdo, por oclusión o estenosis de la arteria cerebelosa posteroinferior, de posible etiología aterotrombótica.

El paciente había acudido cinco días antes a urgencias por mareo y parestesias en la segunda y la tercera rama del trigémino, autolimitada en pocas horas, con exploración neurológica y TAC craneal normal. Se orientó el cuadro como un posible accidente isquémico transitorio, y se le dio el alta sin realizar cambios en el tratamiento antiagregante.

Tras 12 horas de ingreso, el paciente presentaba un aumento de la inestabilidad con vértigo, aumento de la disartria y aparición de disfagia, a lo que se sumó una hemiparesia braquiocrural derecha 4+/5; por ello, se suspendió la antiagregación y se inició la anticoagulación con heparina sódica. En la TAC craneal que se realizó pocas horas después de iniciar la anticoagulación se evidenciaba un hematoma bulbar en la región lateral y posterior izquierda, por lo que se suspendió la anticoagulación, con estabilidad clínica del paciente (Figs. 1b y 1c). Se realizó una ecografía Doppler transcraneal, que fue normal, y de los troncos supraaórticos, que demostró placas en ambas bifurcaciones del 15 al $50 \%$.

En la resonancia magnética (RM) realizada siete días después del ingreso, se objetivó una lesión hemorrágica bulboprotuberancial en la región lateral del bulbo izquierdo (Fig. 2).

En la RM de control realizada un mes después se observaron restos de sangrado en la región del bulbo, con una imagen compatible con cavernoma y drenaje venoso anómalo asociado (Fig. 3).

Actualmente, el paciente presenta, como única secuela, una hemihipoestesia braquiocrural derecha y un aumento de la base de sustentación, y continúa el tratamiento antiagregante con $300 \mathrm{mg} /$ día de aspirina.

Se propuso una exéresis del cavernoma en frío después de un mes del episodio hemorrágico, lo que fue rechazado por el paciente.

\section{DISCUSIÓN}

La hemorragia es, por orden de frecuencia, después de las crisis comiciales, la segunda causa de complicación de los cavernomas 

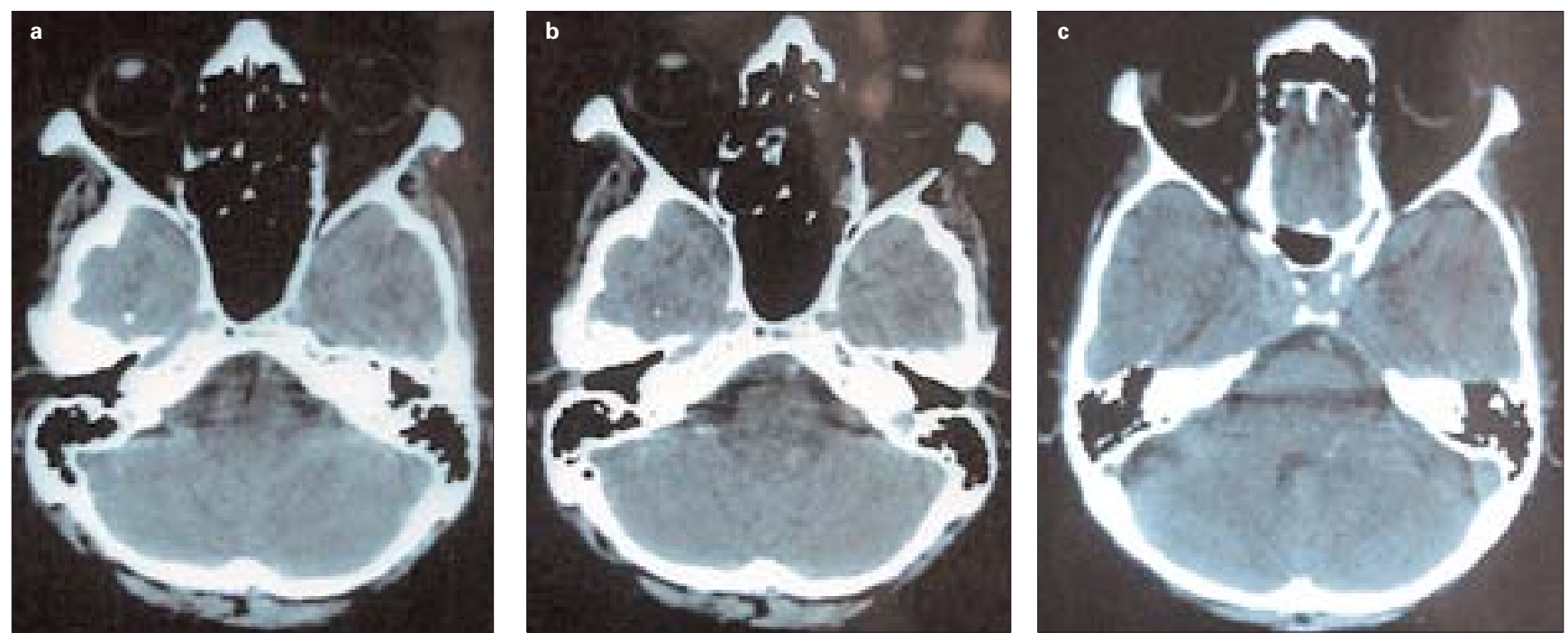

Figura 1. a) TAC del ingreso que es normal; b) y c) se objetiva sangrado en la región lateral bulboprotuberancial izquierda (realizado 12 horas después del ingreso tras iniciar la anticoagulación y tras el empeoramiento clínico)

cerebrales supratentoriales y la primera en la región infratentorial. En el tronco, y por la proximidad de centros elocuentes, como los núcleos de los pares craneales y las vías largas de conducción, es posible que un mínimo efecto de masa o sangrado del cavernoma provoque la aparición de una focalidad neurológica.

En nuestro caso, la focalidad neurológica se manifestó sin evidencia radiológica de sangrado en la TAC. Este tipo de complicación se ha descrito entre el 20 y el 50\% de los casos publicados en revisiones neuroquirúrgicas; el porcentaje más alto fue en el subgrupo correspondiente a los de localización en el tronco. El mecanismo causal puede ser por microsangrados no visibles en la TAC, crecimiento de un pequeño hematoma intralesional o edema perilesional. Pero la explicación más apropiada para justificar la aparición de focalidad sin sangrado evidente lleva implícita conocer la histología y la morfogénesis de los cavernomas [6-8].

Los cavernomas, histológicamente, son una serie de sinusoides vasculares constituidos por una capa de endotelio, en forma trabecular, con un fino tejido de sostén del que prácticamente está ausente el colágeno. Esta masa esponjosa está separada en su parte más externa del resto del parénquima por un tejido conectivo, que en pocas ocasiones está suficientemente engrosado para considerarlo una verdadera cápsula. Los diferentes sinusoides del cavernoma están llenos de sangre, cuyo aporte es muy lento a partir de pequeños vasos de características muy parecidas a las vénulas, aunque en muchas ocasiones se encuentran vasos periféricos al cavernoma de estructura arterial. Este flujo lento de sangre hace particular a estas malformaciones por dos razones: no se visualizan por arteriografía ni por técnicas de evaluación dinámica vascular y el escaso recambio de la sangre de los cavernomas predispone a fenómenos trombóticos intrasinusales. De esta forma, se producen trombosis intracavernoma, con roturas de sinusoides y hemorragias dentro de las trabéculas, lo que conlleva un proceso dinámico continuo dentro del cavernoma, aunque sin cambios en su volumen total. Cuando se rompe este equilibrio, se puede producir un crecimiento del cavernoma sin aparente sangrado, con efecto de masa sobre las estructuras periféricas y aparición de focalidad neurológica, dependiendo de su localización y de la posible afectación de estructuras adyacentes.

En el caso que se describe, el paciente acudió unos días antes a Urgencias por episodios de parestesias faciales y mareo,

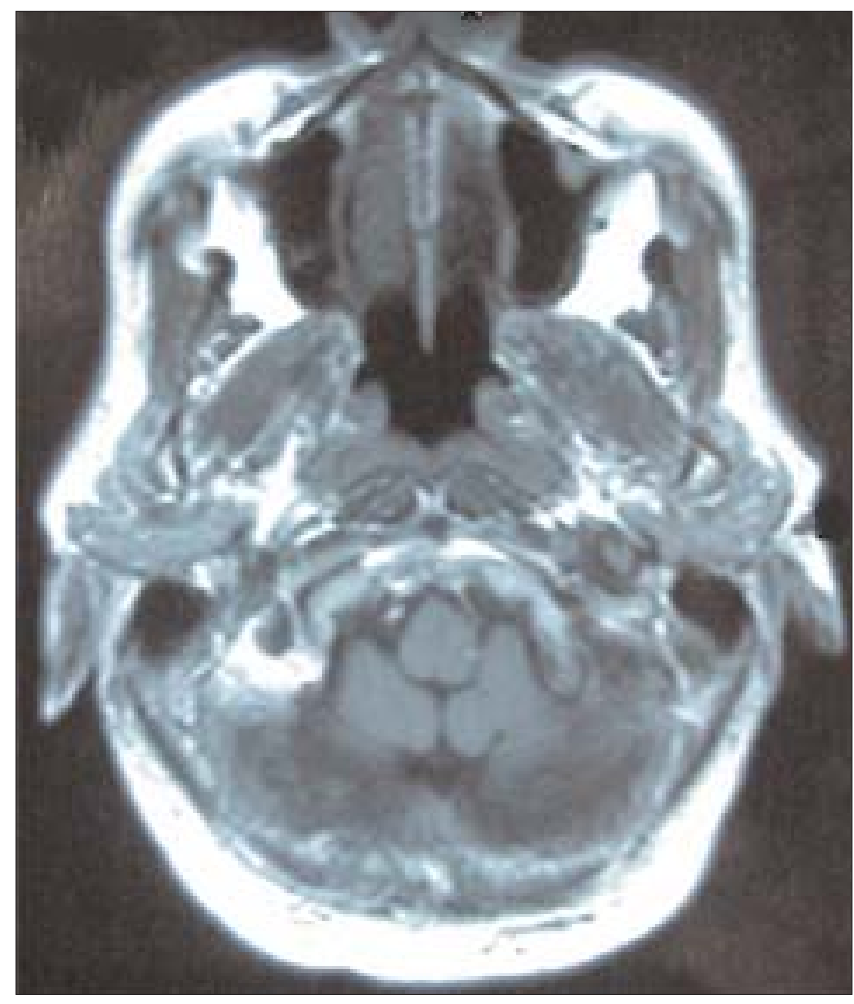

Figura 2. RM donde se observa una hemorragia en la región lateral del bulbo izquierdo.

sin imagen radiológica de sangrado en la TAC. Posiblemente, éste fue el primer momento en que se produjo la rotura del equilibrio dinámico de un cavernoma situado en la porción lateral del bulbo y muy próximo a estructuras elocuentes en la región más inferior del tronco. La clínica posterior, en forma de síndrome de un Wallenberg no es más que la confirmación fidedigna de que el cavernoma está creciendo debido a los fenómenos tromboticohemorrágicos intrasinusoidales, sin que se manifieste radiológicamente el sangrado.

El posterior sangrado del hematoma tras la descoagulación conlleva la duda de si en realidad esta complicación es la conti- 

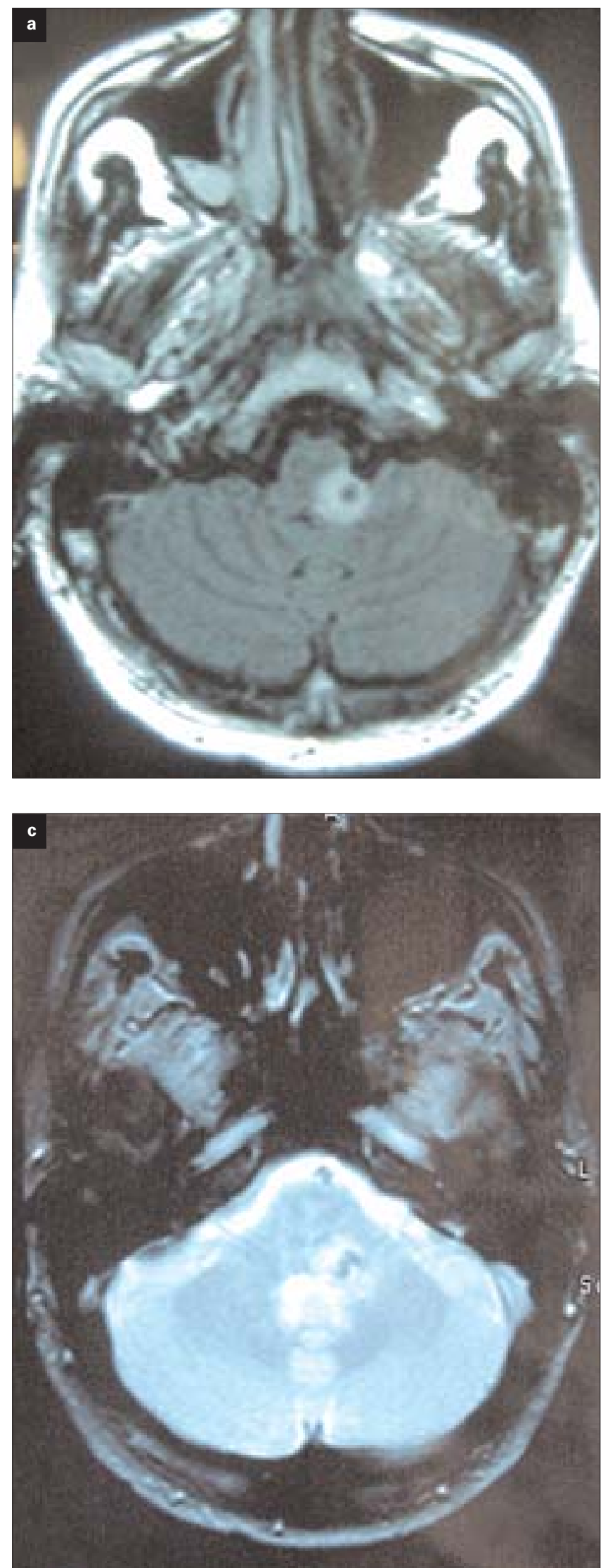

Figura 3. RM en la que se aprecia un cavernoma en esta localización con una imagen serpinginoforme compatible con un drenaje venoso anómalo asociado.

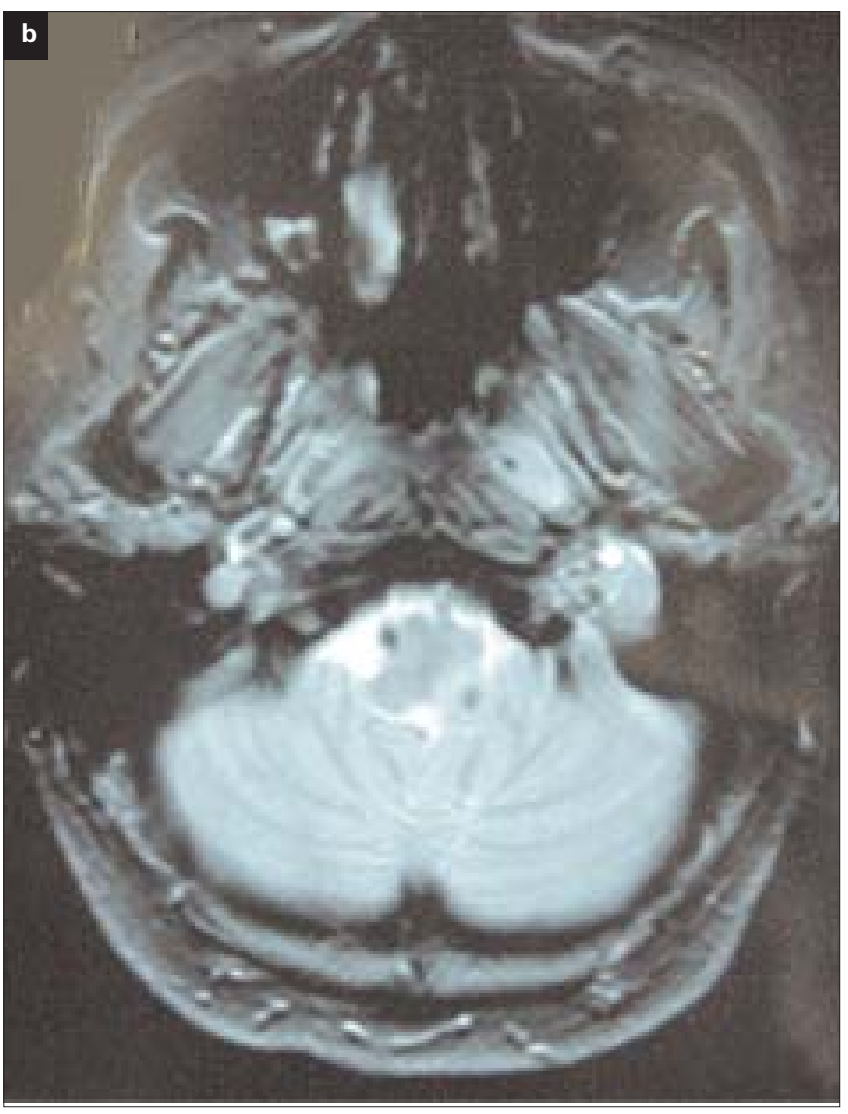

nuación de este proceso dinámico o si ha sido la heparina la causante o el factor que ha ayudado a la formación del hematoma. En nuestro, caso la complicación hemorrágica se supuso que era consecuencia de la heparina y radiología la informó como un infarto hemorrágico.

En este caso, al ser un paciente con varios factores de riesgo cardiovascular, antecedentes de cardiopatía isquémica y estenosis de grandes vasos aortoilíacos, la clínica orientaba a un cuadro de isquemia vertebrobasilar de etiología aterotrombótica. Si bien el Doppler transcraneal en el momento del ingreso podría habernos ayudado a valorar si existían estenosis intravertebrales, no nos sería útil para valorar la permeabilidad de la arteria cerebelosa posteriorinferior (PICA), ya que no se puede aislar correctamente con el Doppler transcraneal. Fue éste el motivo por el que se supuso, hasta que se realizó la segunda RM, que el paciente sufrió un infarto hemorrágico de la PICA izquierda. La realización de la segunda RM se solicitó ante la localización inusual y la cronología en la que apareció la clínica. Los hallazgos eran compatibles con un cavernoma único, con un drenaje venoso anómalo asociado, lo que en ocasiones se ha citado como factor de riesgo para la recurrencia del sangrado. [9-12]

La clínica del síndrome de Wallenberg es meramente anecdótica, y consecuencia de la casualidad de que se sitúe el cavernoma en la localización exacta para ocasionar dicho síndrome. Se han descrito otros síndromes en relación con cavernomas en localizaciones estratégicas, como parkinsonismo, síndromes protuberanciales, e incluso alteración aislada de pares craneales. En nuestro caso, la participación del drenaje venoso en la aparición de la clínica no parece significativa. Muchos de estos cavernomas pasan inadvertidos en las pruebas radiológicas con- 
vencionales de urgencias, como es la TAC, más aún los de fosa posterior; si a esto se suma una clínica o un síndrome poco usual para un cavernoma el diagnóstico se hace sumamente difícil.

Una vez se realizó el diagnóstico del cavernoma, se le planteó al paciente la realización de cirugía esterotáxica, que no aceptó; dicha propuesta se basaba en las series neuroquirúrgicas que apuntan a un alto riesgo de recurrencia de sangrado y morbilidad en las lesiones del territorio vertebrobasilar; pero, los factores de riesgo cardiovascular y la localización la convertían en una intervención de alto riesgo, por lo que, finalmente, no se realizó. No se conoce el riesgo de recurrencia de fenómenos paroxísticos de focalidad neurológica sin evidencia de sangrado provocados por cavernomas. Posiblemente, en la mayoría de estos casos la evolución final será un sangrado, que se confirmará tras la realización de una RM, pero en algunos casos podría tener un curso relativamente indolente, y puede confundirse su diagnóstico con el de esclerosis múltiple, tumores de crecimiento lento, vasculitis o enfermedades mitocondriales.

En conclusión, los angiomas cavernosos, si bien son considerados como malformaciones vasculares de curso benigno, pueden llegar a ser sintomáticos, en especial los del tronco cerebral. Entre las complicaciones de los que se sitúan en esta localización destaca, a diferencia de los supratentoriales, la aparición de focalidad neurológica sin signos de sangrados radiológicos por TAC. Esta complicación clínica es coherente con la teoría de un equilibrio dinámico de sangrados y trombosis de intracavernoma, que en algún momento se rompe y conlleva su crecimiento.

Al igual que otras malformaciones y alteraciones vasculares, como trombosis venosa cerebral, aneurismas, dolicoectasias, etc., los cavernomas pueden mimetizar una focalidad neurológica de etiología aterotromboembólica, e incluso un síndrome vascular bien definido, según su localización y cronopatología.

Aunque la TAC ha sido y sigue siendo la técnica radiológica de urgencia por excelencia, en la actualidad, cuando se instauran tratamientos de fase aguda como la trombólisis, o en situaciones en las que progresa la clínica neurológica y se inicia la heparina, la RM podría plantearse como la técnica de imagen más fiable en la selección y en los cambios de decisiones terapéuticas.

Los cavernomas, después del sangrado, suelen tener una baja recurrencia de sangrado, pero la localización en el tronco conlleva, en caso de sangrado, mayor expresión clínica y mayores secuelas. Por esta razón, algunos cirujanos los consideran de riesgo suficientemente alto como para ser candidatos a cirugía, si no existe contraindicación para ella.

\section{BIBLIOGRAFÍA}

1. Kupersmith MJ, Kalish H, Epstein F. Natural history of brainstem cavernous malformations. Neurosurgery 2001; 48: 47-54.

2. Maeder P, Gudinchet F, Meuli R, De Tribolet N. Development of a cavernous malformation of the brain. AJNR Am J Neuroradiol 1998; 19: 1141-5.

3. Zameel Cader M, Winer JB. Cavernous haemangioma mimicking multiple sclerosis. Br Med J 1999; 318: 1604-5.

4. Marc Simard J, García Bengochea F. Cavernous angioma: a review of 126 collected and 12 new clinical cases. Neurosurgery 1986; 18: 162-71.

5. Steigner HJ, Marrkwalder TM. Clinicopatological relations of cerebral cavernous angiomas: observations in eleven cases. Neurosurgery 1987; 21: 879-83.

6. Wong JH, Awad IA, Kim JH. Ultrastructural pathological features of cerebrovascular malformations: a preliminary report. Neurosurgery 2000; 46: 1454-9.

\section{SÍNDROME DE WALLENBERG COMO FORMA \\ DE PRESENTACIÓN DE UN ANGIOMA CAVERNOSO}

Resumen. Introducción. Los angiomas cavernosos representan el $5-13 \%$ del total de malformaciones vasculares. En el $75 \%$ de los casos se encuentran en la fosa posterior, y hasta en el 30\%, asociado a un drenaje venoso anómalo. La principal complicación son las hemorragias; es excepcional la presencia de focalidad neurológica sin evidencia radiológica de sangrado. Caso clínico. Presentamos un caso de un varón de 54 años con factores de riesgo cardiovascular, que muestra un cuadro progresivo en 72 horas de duración de afectación de los pares craneales bajos izquierdos, déficit sensitivo y alteración de la coordinación compatible con un síndrome de Wallenberg. Con la tomografía axial computarizada craneal en dos ocasiones normal, se realizó la orientación diagnóstica de ictus vertebrobasilar en progresión. Cinco días después, la resonancia magnética (RM) demostró la presencia de un angioma venoso y un drenaje venoso anómalo asociado. Conclusión. Los angiomas cavernosos presentan un equilibrio dinámico de sangrado y trombosis de intracavernoma, con un flujo venoso muy lento. Una rotura en este equilibrio supone un aumento de la presión de intracavernoma y la afectación del tejido circundante, sin expresión radiológica de sangrado. La RM ayuda en estos casos a diferenciar una malformación vascular de flujo lento de un cuadro ictal isquémico de origen arterial. [REV NEUROL 2004; 39: 837-40]

Palabras clave. Angiomas cavernosos. Angiomas venosos. Drenaje venoso anómalo. Ictus. Wallenberg.
7. Cantore G, Missori P. Cavernous angiomas of the brain stem. Intra-axial anatomical pitfalls and surgical strategies. Surg Neurol 1999; 52: 84-94.

8. Mahla K, Rizk T. Intracranial cavernoma. Surgical results of 47 cases. Neurochirurgie 1999; 45: 286-92.

9. Pozzati E, Giuliani G, Nuzzo G, Poppi M. The growth of cerebral cavernous angiomas. Neurosurgery $1989 ; 25$ : $92-7$.

10. Sandal Cioglu E, Wiedemayer H. Surgical removal of brain stem cavernous malformations: surgical indications, technical considerations, and results. J Neurol Neurosurg Psychiatry 2002; 72: 351-5.

11. Abdulrauf SI, Kaynar MY. A comparison of the clinical profile of cavernous malformations with and without associated venous malformations. Neurosurgery 1999; 44: 41-6.

12. Del Curling TB. The natural history of intracranial venous angiomas. J Neurosurg 1991; 75: 715-22.

\section{SÍNDROMA DE WALLENBERG COMO FORMA DE APRESENTAÇÃO DE UM ANGIOMA CAVERNOSO}

Resumo. Introdução. Os angiomas cavernosos representam 5-13\% do total das malformações vasculares. Em $75 \%$ dos casos encontram-se na fossa posterior, e até $30 \%$ associado a drenagem venosa anormal. A principal complicação é constituída pelas hemorragias; é excepcional a presença de focalidade neurológica sem evidência radiológica de hemorragia. Caso clínico. Apresentamos um caso de um homem de 54 anos de idade com factores de risco cardiovascular, que apresenta um quadro progressivo, de 72 horas de duração, com envolvimento dos pares cranianos baixos esquerdos, défice sensitivo e alteração da coordenação compatível com um síndroma de Wallenberg. Com tomografia axial computorizada craniana normal em duas ocasiões, fez se o diagnóstico de AVC vertebrobasilar em progressão. Após cinco dias, a ressonância magnética (RM) mostrou a presença de um angioma venoso e uma drenagem venosa anómala associada. Conclusão. Os angiomas cavernosos apresentam um equilíbrio dinâmico de hemorragia e trombose de intra-cavernoma, com um fluxo venoso muito lento. Uma ruptura deste equilíbrio supõe um aumento de pressão de intra-cavernoma e o envolvimento do tecido circundante, sem expressão radiológica de hemorragia. A RM ajuda nestes casos a diferenciar uma malformação vascular de fluxo lento de um quadro de AVC isquémico de origem arterial. [REV NEUROL 2004; 39: 837-40]

Palavras chave. Angiomas cavernosos. Angiomas venosos. AVC. Drenagem venosa anómala. Wallenberg. 